\title{
GAI - distinct genotype and phenotype characteristics in reported Slovak patients
}

\author{
Lisyova $\mathrm{J}^{1}$, Petrovic $\mathrm{R}^{1}$, Jurickova $\mathrm{K}^{2}$, Brennerova $\mathrm{K}^{3}$, Urbanova $\mathrm{D}^{4}$, Behulova $\mathrm{D}^{5}, \mathrm{Bzduch}^{3}$, Chandoga $\mathrm{J}^{1}$ \\ Institute of Medical Biology, Genetics and Clinical Genetics, Comenius University Faculty of Medicine and University Hospital, \\ Department of Molecular and Biochemical Genetics - Centre of Rare Genetic Diseases, Bratislava, Slovakia. lisyova.j@gmail.com
}

\section{ABSTRACT}

OBJECTIVES: The clinical, biochemical and genetic findings in two Slovak patients with glutaric aciduria type I (GAI) are presented.

BACKGROUND: GAI is a rare autosomal recessive neuro-metabolic disorder caused by deficiency of glutarylCoA dehydrogenase, which is involved in the catabolic pathways of lysine, hydroxylysine and tryptophan. This enzymatic defect gives rise to elevated levels of glutaric acid (GA), 3-hydroxyglutaric acid (3-OH-GA) and glutarylcarnitine (C5DC) in body fluids.

METHODS: Biochemical and molecular-genetic tests were performed. Urinary organic acids were analysed by Gas Chromatography/Mass Spectrometry (GC/MS) and the entire coding region of the GCDH gene, including flanking parts, was sequenced.

RESULTS: We found the presence of typical metabolic profile and novel causal pathogenic variants in both GAI patients. CONCLUSION: We present the first report of two Slovak patients with GAI, which differed in the clinical and biochemical phenotype significantly. They were diagnosed by two distinct approaches - selective and newborn screening. Their diagnosis was complexly confirmed by biochemical and later on molecular-genetic examinations. Though we agreed with a thesis that early diagnostics might positively influenced patient's health outcome, contradictory facts should be considered. Supposed extremely low prevalence of GAI patients in the general population and/or the existence of asymptomatic individuals with a questionable benefit of the applied therapeutic intervention for them lead to doubts whether the inclusion of disease into the newborn screening programme is justified well enough (Tab. 1, Fig. 3, Ref. 41). Text in PDF www.elis.sk.

KEY WORDS: glutaric aciduria type I, glutaric acid, 3-hydroxyglutaric acid, GCDH gene, novel pathogenic variants, newborn screening.

\begin{abstract}
Abbreviations: ALT - alanine transaminase, AST - aspartate transaminase, 2-OH-GA - 2-hydroxyglutaric acid, 3-OH-GA 3-hydroxyglutaric acid, C5DC - glutarylcarnitine, C8 - octanoylcarnitine, C16 - hexadecanoylcarnitine, ETF - electron-transfer flavoprotein, FAD - flavin adenine dinucleotide, GA - glutaric acid, GAI - glutaric aciduria type I, GCDH - glutaryl-CoA de-

${ }^{1}$ Institute of Medical Biology, Genetics and Clinical Genetics, Comenius University Faculty of Medicine and University Hospital, Department of Molecular and Biochemical Genetics - Centre of Rare Genetic Diseases, Bratislava, Slovakia, ${ }^{2} 2$ nd Department of Pediatrics, Comenius University Children's Hospital, Centre for inborn errors of metabolism, Bratislava, Slovakia, ${ }^{3} 1$ st Department of Pediatrics, Comenius University Children's Hospital, Bratislava, Slovakia, ${ }^{4}$ Department of Neonatology and Intensive Medicine, Comenius University Faculty of Medicine and University Children's Hospital, Bratislava, Slovakia, and ${ }^{5}$ Department of Laboratory Medicine, University Children's Hospital, Centre for inborn errors of metabolism, Bratislava, Slovakia
\end{abstract}

Address for correspondence: J. Lisyová, MD, Institute of Medical Biology, Genetics and Clinical Genetics, Comenius University Faculty of Medicine and University Hospital, Department of Molecular and Biochemical Genetics - Centre of Rare Genetic Diseases, Mickiewiczova 13, SK-813 69 Bratislava, Slovakia.

Phone: +421.2 .57290192$

Acknowledgements: The authors also would like to acknowledge the Newborn Screening Centre of SR for providing information about biochemical findings from newborn screening. hydrogenase, GCDH - gene encoding glutaryl-CoA dehydrogenase, GC/MS - gas chromatography/mass spectrometry, MCAD - medium-chain acyl-CoA dehydrogenase, MS/MS - tandem mass spectrometry, ESI-MS/MS - electrospray-ionisation tandem mass spectrometry, MRI - magnetic resonance imaging, NBS - newborn screening, NNSC - National Newborn Screening Centre, PCR polymerase chain reaction, USG - ultrasonography

\section{Introduction}

Glutaric aciduria type I (GAI, synonym glutaric acidemia type I, OMIM\#231670) is a rare organic aciduria with autosomal recessive inheritance caused by deficiency of glutaryl-CoA dehydrogenase (GCDH, EC 1.3.8.6). This inborn error of metabolism has been firstly described in 1975 by Goodman in two patients (1). Actually more than 500 cases have been reported worldwide.

The responsible GCDH gene (OMIM*608801) is localised on chromosome 19 (19p13.2) (2), spans approximately $7 \mathrm{~kb}$ and contains 12 exons, but the first exon is noncoding. GCDH gene encodes a flavin adenine dinucleotide (FAD)-dependent mitochondrial matrix flavoprotein, which catalyses dehydrogenation and decarboxylation of glutaryl-CoA to crotonyl-CoA in the common degradative pathway of L-lysine, L-hydroxylysine and Ltryptophan $(3,4)$. According to published sources, more than 200 


\section{1-638}

pathogenic variants were described in the GCDH gene, most of them are point mutations (missense/nonsense type) $(5,6)$.

The presence of serious pathogenic variants in the $G C D H$ gene leads to no residual enzyme activity and consequently to the characteristic metabolic pattern. On the other hand, less serious pathogenic variants preserve a sufficient residual enzyme activity and therefore the excretion of glutaric acid (GA) can be elevated only mildly or can be even normal $(7,8)$. Based on biochemical findings, the amount of excreted GA and enzyme activities, two subgroups of GAI patients can be distinguished. Low excretors are with the residual enzyme activity up to $30 \%$ and are defined by a lower level of urinary GA excretion (below $100 \mathrm{mmol} / \mathrm{mol}$ creatinine) and high excretors with a complete loss of enzyme activity typically excrete high amounts of GA usually above 100 $\mathrm{mmol} / \mathrm{mol}$ creatinine (9). GCDH genotype predicts only the biochemical (not clinical) phenotype and high or low excretors may have the risk of developing symptoms linked to this disorder.

GAI is clinically characterised by a progressive movement disorder such as dystonia and dyskinesia. The age of onset is typically within the first three years of life, but can range from the prenatal period of foetus to adulthood (10). Early clinical diagnosis is hampered by the lack of characteristic or even pathognomonic signs and symptoms before an encephalopathic crisis (6). After the birth, there is usually a period of approximately one year of apparently normal development in children with GAI or only mild neurological symptoms such as irritability and jitteriness may be present. Moreover, macrocephaly represents a common though nonspecific symptom found in $75 \%$ of cases and appears shortly after birth (11). Motor delay, hypotonia, dystonia, and dyskinesia can progressively develop during the first years of life. Although the intellect and cognitive functions are relatively well-preserved in GAI patients, the numerous structural and functional cerebral abnormalities that can begin in the last trimester of pregnancy had been found. The white matter changes seem to increase with age, both in patients with high as well as low excretion phenotypes (12). The suspicion of GAI may be often omitted in patients with cerebral palsy and mental retardation as isolated symptoms $(13,14)$. Due to cortical atrophy and expanded cerebrospinal fluid spaces causing stretching of the cortical veins, GAI patients with typical brain abnormalities on MRI have also an increased risk for subdural haematomas after less significant trauma compared to the healthy individuals. Based on the latest data, their incidence in GAI patients was assessed at $4 \%$, which is lower than compared to $20-30 \%$ reported previously (15).

The first clinical manifestation of the disease may occur as an acute encephalopathic episode triggered by infection, immunisation or surgical intervention and leads to the sudden onset of hypotonia and loss of head control as a first indicator of GAI (16). In undiagnosed and untreated patients, the neurological impairment can develop in age of 3-36 months of life. An irreversible movement disorder develops in approximately $80-95 \%$ of untreated patients (17). The first clinical manifestation can cause the encephalopathy and irreversible brain damage, therefore the recognition of macrocephaly in utero if possible and subsequent early diagnostics and treatment is essential (18). According to data collected by Heringer et al (19), in the group of 176 GAI patients, they detected significantly less frequent occurrence of the movement disorders in patients identified by newborn screening (NBS) compared to patients revealed by selective screening ( $26 \%$ vs $73 \%$ ). The median age at diagnostics from NBS was 11 days of life, however in later-onset patients from selective screening the diagnosis was established in median age of 450 days of life. The initiation of early treatment before the symptoms occur is important and can prevent the irreversible brain damage and even death.

\section{Material and methods}

\section{Samples}

This study was conducted in two GAI patients, urine, serum and whole blood samples from patients for DNA and biochemical analyses were provided. Also the blood samples of parents were received for confirmation of their carrier status.

\section{Metabolite analyses}

Routine blood and urine tests were performed for evaluation of liver and renal function, serum electrolytes and glucose. The urinary organic acids were extracted by ethyl acetate, derivatised and analysed by GC/MS using the Thermo ITQ900 analyser (20).

\section{Molecular-genetic analysis}

Genomic DNA was extracted from peripheral blood leukocytes using NucleoSpin ${ }^{\circledR}$ Blood (Macherey-Nagel). The entire coding sequence of the GCDH gene was amplified by polymerase chain reaction (PCR) using 10 pairs of primers designed by our laboratory using Primer3 (Tab. 1). The optimised reaction cycling conditions were as followed: initial denaturation at $95{ }^{\circ} \mathrm{C}$ for 3 minutes, 30

Tab. 1. The primer sequences designed for the amplification of the coding region of GCDH gene.

\begin{tabular}{ll}
\hline GCDH gene & primer sequence (5' $\left.\rightarrow 3^{\prime}\right)$ \\
\hline GCDH_ex2+3F & ACTGTAGCCTCGGCAGTGAA \\
GCDH_ex2+3R & TCTCGGATTCTGGGACAGAG \\
\hline GCDH_ex4F & CACCTGATCAGTCTCGCTTG \\
GCDH_ex4R & GAAAAACTGCAAAGGGACCA \\
\hline GCDH_ex5F & AGAGACTCATGCCTCGCATC \\
GCDH_ex5R & CAGAGGGTTCTGCAGTGTGT \\
\hline GCDH_ex6F & CTGCCTCCTTGTGTGTCCTT \\
GCDH_ex6R & CAGAATAGGCTCAGGGGACA \\
\hline GCDH_ex7F & GGCAGCCTTGTGACTTTGTC \\
GCDH_ex7R & AGTCGGTGAGGGGTCTGAC \\
\hline GCDH_ex8F & GGAGACCAGAGCCCACTACA \\
GCDH_ex8R & CCGGCTGAGTAAGAATCACC \\
\hline GCDH_ex9F & GCCATCTGTGATGTGAACCA \\
GCDH_ex9R & AGGACGTCACTGGTCATTCC \\
\hline GCDH_ex10F & AAGCTTGGGGGCACTGAG \\
GCDH_ex10R & AGGACAAGAGGGACAGCAGA \\
\hline GCDH_ex11F & GTCCCTCATTGGGAGCTTG \\
GCDH_ex11R & TGGAGTTGGACTCAGACCTGT \\
\hline GCDH_ex12F & CAGTGGCCTGGGGATACAT \\
GCDH_ex12R & TCCATGGTTAAAAGTGGATTGA \\
\hline
\end{tabular}


cycles consisting of denaturation at $95^{\circ} \mathrm{C}$ for 30 seconds, annealing at $62{ }^{\circ} \mathrm{C}$ (exons $2-6,8-11$ ), $59^{\circ} \mathrm{C}$ (exon 7 ) and $58^{\circ} \mathrm{C}$ (exon 12) for 30 seconds and extension at $72{ }^{\circ} \mathrm{C}$ for 1 minute, followed by the final extension step at $72{ }^{\circ} \mathrm{C}$ for 5 minutes. Purified PCR products were sequenced using the ABI 3100 Avant Genetic Analyzer. Mutation analysis was performed using SeqScape ${ }^{\circledR}$ Software v2.6. All identified pathogenic variants were confirmed by sequencing of both DNA strands and confirmed in the parents.

\section{Results}

Case 1

The female patient from the first at risk pregnancy of healthy consanguineous parents was delivered in the 40th gestational week by the caesarean section, because of macrocephaly (birth length $52 \mathrm{~cm}$ and weight $3550 \mathrm{~g}$ ). The girl was monitored from the age of two months by a neurologist for suspicion of demyelinating disorder. USG examination of the brain in 1 year of age showed no pathological finding. In psychomotor development only speech difficulties and delays were observed. In the third year of life, an intermittent altered state of consciousness (from somnolence to sopor) and dystonic attacks were presented during an acute gastroenteritis of rotavirus aetiology. In the laboratory tests, severe uncompensated metabolic acidosis ( $\mathrm{pH} 7.26)$, hypoglycaemia (1.4 mmol. $\left.1^{-1}\right)$, hyperuricaemia $\left(630 \mu \mathrm{mol} .1^{-1}\right)$ and mildly elevated liver transaminases (activity of AST $2.57 \mu \mathrm{kat} . \mathrm{l}^{-1}$ and ALT $1.04 \mu \mathrm{kat} . \mathrm{l}^{-1}$ ) were found. Patient was treated by standard parenteral hydration therapy, but the clinical recovery had been very slow (4 weeks). During this period, she lost the ability to walk, talk and swallow. Because of clinical picture and persistent metabolic acidosis with significantly decreased level of free carnitine $\left(3.3 \mu \mathrm{mol} . \mathrm{l}^{-1}\right.$, reference range 22.2-52.2 $\mu \mathrm{mol} .1^{-1}$ ) a suspicion for inherited metabolic disease was made. An analysis of urinary organic acid in the Institute of Inherited Metabolic Disorders in Prague was performed, where increased levels of GA and 3-hydroxyglutaric acid (3-OHGA) were detected.

The course of the disease had been complicated during an acute relapsing pyelonefritis and later on by skin abscesses, which required immunomodulating therapy. In time of infectious illness, the level of GA increased rapidly $(818-5300 \mathrm{mmol} / \mathrm{mol}$ creatinine, reference $<5 \mathrm{mmol} / \mathrm{mol}$ creatinine) (Fig. 1). During an acute attack period, the patient was treated by riboflavine, L-carnitine and with low-lysine diet, and in the compensated state of disorder only L-carnitine was applied. After the administration of $1 \mathrm{~g} /$ day of L-carnitine the level of carnitine have been normalised or even increased (from 34.2 to $82.0 \mu \mathrm{mol} . \mathrm{I}^{-1}$ ). Also the monitoring of the
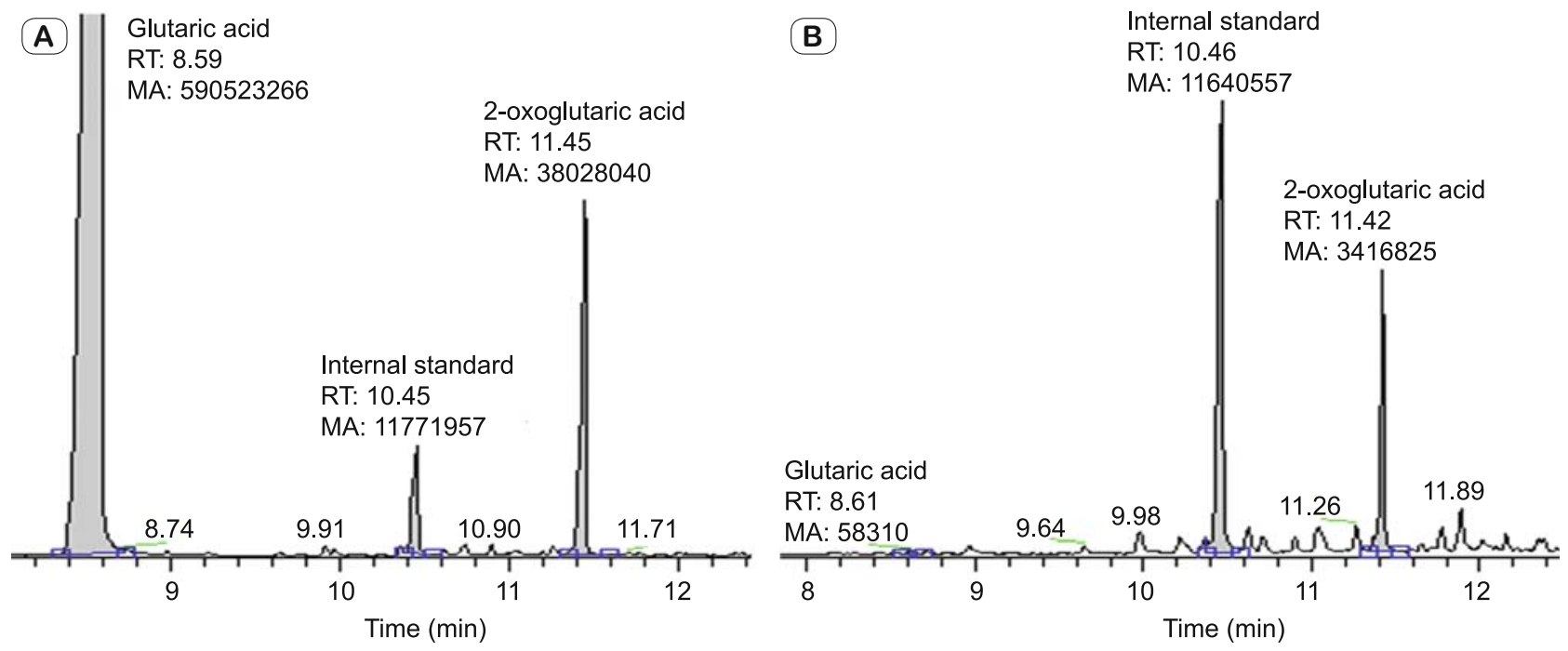

C
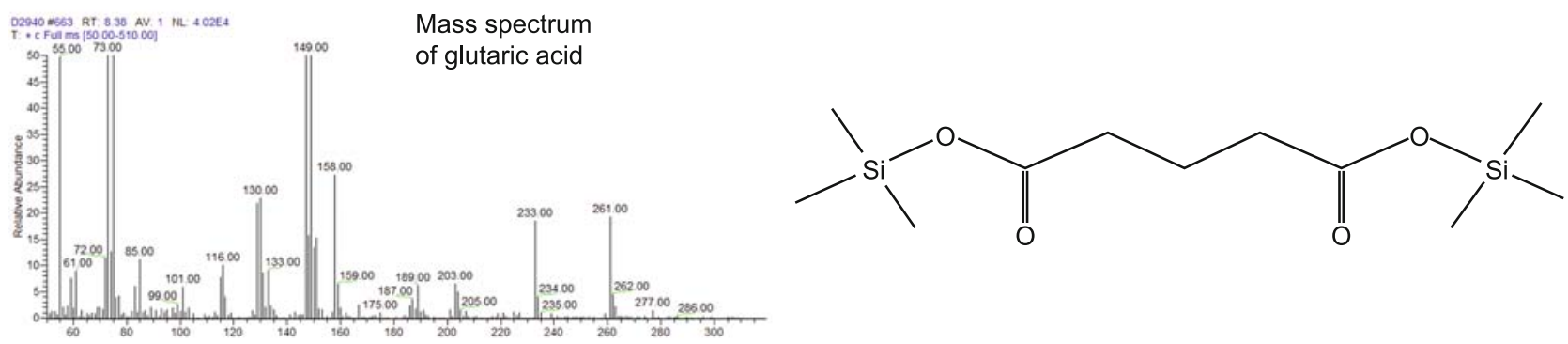

Fig. 1. Profile of urinary organic acids by GC/MS in GAI patient in attack - grossly elevated levels of GA and elevated level of 2-oxoglutaric acid (A), control urine (B) and mass spectrum and chemical formula of GA-2TMS (C). 


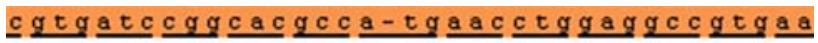

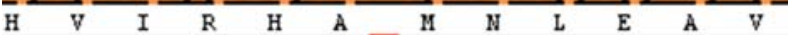

C GTGATCCGGCACGCCAATGAAC C T G A G G C G T GA A T GATC C G G C A C G C CAAT GAAC C T G GA G G C C G T GA A (A)

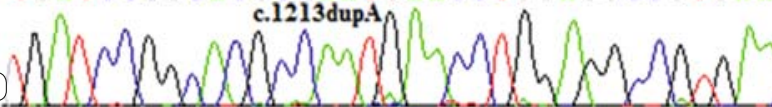
C GTGATCCGGCACGCCA-UKRAMCYKGRRG $\$$ C $\$ K K R A$ GT GATC C G C A C G C C A UKRAMCYKGRRGSCSKKRA

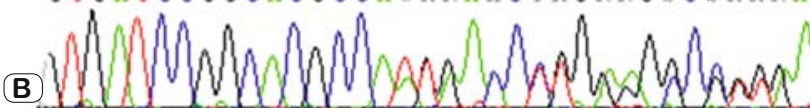
C GTGATCCGGCACGCCA-UKRAMCYKGRRG SCSKKRA GT GATCC G G A C G C CA UKRAMCYKGRRG S C SKRRA

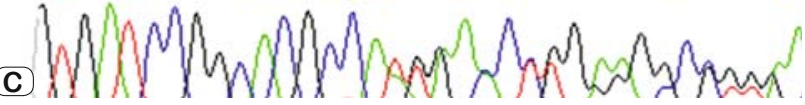

Fig. 2. Sequencing analysis of exon 11 of the GCDH gene. $A-c .1213 d u p A$ in homozygous state found in patient no.1, $B$ and $C-c .1213 d u p A$ in heterozygous state detected in both parents ( $\mathrm{B}$ - mother, $\mathrm{C}$ - father).

GA level indicated the efficiency of therapy $(176-298 \mathrm{mmol} / \mathrm{mol}$ creatinine). Thus a long-term treatment with carnitine supplementation had protected the patient from the occurrence of encephalopathic crises for the following twenty years. On MRI examination, supra and infratentorial demyelinating lesions were detected.

The patient is continuously monitored, well compensated without dystonia, atetotic movements and she is fully mobile. She has achieved secondary education and only in the case of disturbance she suffers from stammering (balbuties).

\section{Molecular-genetic findings in case 1}

In the 24-years-old female patient we had found the frameshift mutation c.1213dupA in the exon 11 of the GCDH gene in the homozygous state. This novel duplicating insertion is assumed to be pathogenic due to altering the open reading frame and leading to the formation of premature stop codon after 14 missense amino acids and to the truncation of the peptide (p.Met405Asnfs*14). The heterozygous carrier status for c.1213dupA in both parents was confirmed (Fig. 2).

\section{Case 2}

The boy from the first physiological gravidity, delivered in the 39th gestational week with birth length $50 \mathrm{~cm}$, weight 3180 $\mathrm{g}$ and Apgar score of 10 was detected by positive newborn screening examination of glutarylcarnitine (C5DC). Concentration of C5DC and ratios of other acylcarnitines from dried blood spot according to NNSC were $2.12 \mu \mathrm{mol} .1^{-1}$ (reference 0-0.3 $\mu \mathrm{mol} . \mathrm{l}^{-1}$ ), ratios $\mathrm{C} 5 \mathrm{DC} / \mathrm{C} 161.05$ (reference $0-0.35$ ) and $\mathrm{C} 5 \mathrm{DC} / \mathrm{C} 8$ 69,75 (reference 0-5.8). Based on the newborn screening results, the eutrophic newborn was admitted to hospital to the regional recall centre because of high suspicion of GAI. Elevated levels of GA $202.8 \mathrm{mmol} / \mathrm{mol}$ creatinine, 3-OH-GA $19.4 \mathrm{mmol} / \mathrm{mol}$ creatinine (reference $<3$ ) and decreased level of carnitine $6.9 \mu \mathrm{mol} . \mathrm{l}^{-1}$ had proved the diagnosis supposed by NBS.

Chosen therapeutic approach was based on summarised guidelines by Heringer et al (21). The authors had been emphasised that the early therapeutic intervention prevents the development of striatal injury and resulting neurological symptoms in the majority of asymptomatic patients identified in the neonatal period by NBS. In spite of this fact, MRI examination of the brain revealed the frequent occurrence of extrastriatal abnormalities with unclear relevance in these children which may manifest clinically later on. Therefore, the main aim of NBS is to identify these patients early and initiate the treatment in asymptomatic stage in order to positively influence their clinical outcome.

Our clinically asymptomatic patient was monitored and treated from 10th day of life. The neurological examination including USG of the brain did not detect any positive findings. The original specific dietary regimen was based on the restriction of the lysine intake and was set at $100 \mathrm{mg} / \mathrm{kg} / \mathrm{day}$. Because of detection of low level of lysine in blood the lysine dosage was increased to $108 \mathrm{mg} / \mathrm{kg} /$ day. L-carnitine intake was adjusted to $70 \mathrm{mg} / \mathrm{kg} /$ day. A patient was discharged in good condition to home aftercare, but he is still continuously monitored in the metabolic department.

\section{Molecular-genetic findings in case 2}

In the newborn male patient, we have detected the pathogenic variant c. $1262 \mathrm{C}>\mathrm{T}$ (rs121434367) in the exon 12 of the GCDH gene in the heterozygous status, which caused an exchange from alanine to valine at amino acid position 421 in the precursor protein (p.Ala421Val). The second sequence variant was the substitution c. $1225 \mathrm{G}>\mathrm{A}$ in the exon 11 of the $G C D H$ gene in the heterozygous state causing an exchange from alanine to threonine at amino acid position 409 in the precursor protein (p.Ala409Thr). This sequence variant has not been reported until now. The carrier status for identified variants in both parents was detected. The mother is a carrier for unreported variant c. $1225 \mathrm{G}>\mathrm{A}$ and the father is a carrier of a second substitution c. $1262 \mathrm{C}>\mathrm{T}$ both in the heterozygous state (Fig. 3).

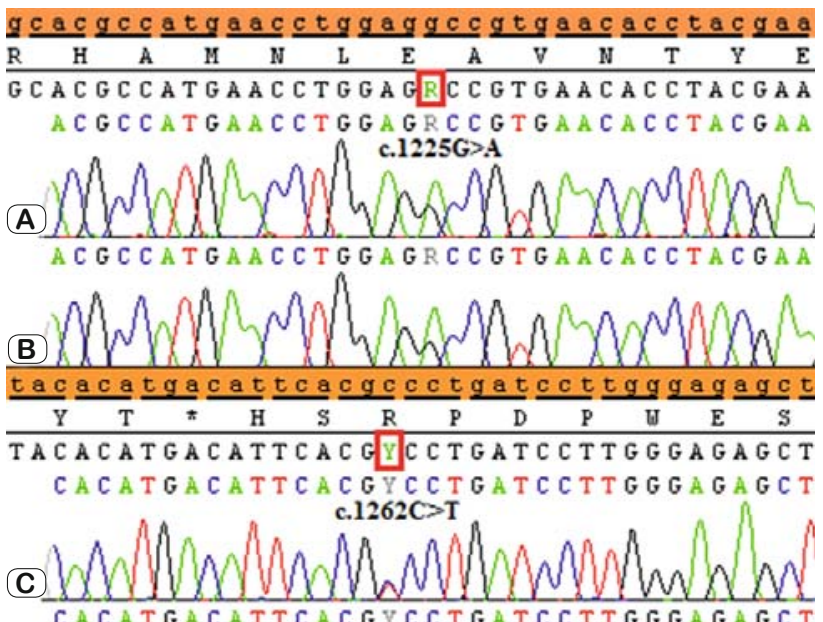
CACATGACATTCACGYCCTGATCCTTGGGAGAGCT

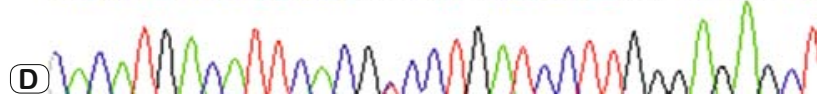

Fig. 3. Sequence analysis of exon 11 and 12 of the GCDH gene. A and $B$ - c.1225G $>$ A sequence variant in heterozygous state in patient 2 (A) and his mother (B), C and D - pathogenic mutation c.1262C $>\mathrm{T}$ in heterozygous state in patient 2 (C) and his father (D). 
In order to predict the effect of c.1225G $>$ A (p.Ala409Thr) missense variant on GCDH function prediction software MutationTaster was used. This sequence variant was supposed to be a probably deleterious. A substitution from hydrophobic alanine residue to polar threonine, which may participate in the hydrogen bonds formation can probably affect the GCDH function.

\section{Discussion}

GAI represents a rare treatable disease when diagnosed early after birth with estimated prevalence based on neonatal screening programmes in Germany, Australia and USA of 1: 106900 newborns, but this can vary in different populations or in other ethnic groups (22). Based on the current natality in Slovakia and taking into account the prevalence in other European countries, we could say that approximately no more than one patient per two years could be revealed. Nevertheless, during a period of last twenty years, only three patients with GAI had been diagnosed in Slovakia. However, the complete clinical, laboratory and molecular-genetic data are available only in two of them.

In the recent study we reported these two patients diagnosed by diverse approaches - selective and newborn screening. We refer substantially distinct health outcome probably due to the different age at diagnostics, initiation of treatment and type of detected mutations. A first female patient was diagnosed at the age of three years based on severe clinical presentation developed after gastroenteritis. Unfortunately, macrocephaly as a typical symptom for GAI, which had been presented even before birth and hypotonic features remained unnoticed before the encephalopathic crisis occurred. At the time of disease onset it was not possible to provide laboratory diagnostics of organic acidurias in Slovakia. But extremely low levels of free carnitine in this patient led to the suspicion of metabolic disorder, which was subsequently confirmed abroad. The second male patient was diagnosed in the neonatal period by NBS programme.

In past, particularly the patients without typical clinical symptoms (macrocephaly, developmental delay and neurological impairment) could remain undiagnosed. From this point of view, the diagnostic approach based on NBS is beneficial. The experience with NBS in other countries showed that the majority of newborns are asymptomatic though a macrocephaly and neurological symptoms such as hypotonia and asymmetric posturing are often present (23). Therefore, the NBS is even more important in a high-risk population such as: the Amish Community in Pennsylvania (USA), the original inhabitants of the Canadian province of Ontario and Manitoba called Oji-Cree (Canada), the Irish travellers (U.K.), the Lumbee tribe in North Carolina (USA) and the indigenous inhabitants of South Africa (17), where the prevalence of GAI should be up to 1: 300 . The occurrence of GAI in state of Andhra Pradesh in India appears to be relatively more frequent caused by a higher rate of consanguinity and endogamous marriages (18).

On the other hand, it is important to mention that cases of GAI with adult-onset exist, too. Interestingly, the clinical outcome in documented patients is dramatically different from those with the typical early-onset. The phenotypic heterogeneity between early-onset and late-onset disease is probably caused by potential existence of additional acquired, genetic and/or environmental factors. The reported late-onset signs include recurrent headaches/migraine, oculomotor symptoms, neurologic and cognitive deterioration, leukoencephalopathy, lower extremities weakness and numbness, hyporeflexia, paresthesias, spasticity, gait disturbances, incontinence, bipolar disorder, axonal and demyelinating neuropathy (24-28). Moreover, GCDH pathogenic variants known to be associated with the severe early-onset disease were found in patients with mild adult-onset or even asymptomatic, too $(27,29)$. It is also important to mention that up to $25 \%$ of undiagnosed (and also untreated) patients remain asymptomatic (15).

The first studies concerning inclusion of GAI into the newborn screening programmes were initiated in the late 1990s in Australia, Germany and certain U.S. states. Since GAI meets several fundamental criteria for inclusion in the NBS programme such as the screened disease should be serious or potentially serious, should be treatable and performed test must be sensitive and costeffective, in present represents a part of newborn screening programmes worldwide for more than fifteen years. In spite of that, GAI is currently incorporated into the NBS panel only in one third of E.U. member states. GAI is also not incorporated into NBS in most regions of China and the selective screening is applied (30). If NBS programmes are not available, the majority of patients are identified after the first disease attack and the treatment is delayed.

Since 2013, the screening of GAI was officially included also in the Slovak newborn screening programme. In the past, selective metabolic screening was performed by GC/MS and analysis of urine organic acids was principal approach for detection of GAI patients. Nowadays, the newborn screening test is based on the determination of C5DC concentrations in dried blood spots by tandem Mass Spectrometry (MS/MS) method and was shown to be cost-effective. Equally, Bessey et al (31) had assessed the cost-effectiveness of expanded NBS programme in England. Their results suggested that expanded NBS including GAI was potentially cost-saving in the meaning of lower total costs and higher quality of life compared to no screening. Although the sensitivity of C5DC screening in a general population represents almost $95 \%$ (32), the cases of missed low excretors are reported.

Biochemically, GAI is characterised by the accumulation of glutaric acid (GA), 3-hydroxyglutaric acid (3-OH-GA), glutaconic acid (not obligatorily) and glutarylcarnitine (C5DC) in the body fluids (urine, blood, cerebrospinal fluid) and tissues (33). These metabolites can be analysed by Gas Chromatography/Mass Spectrometry (GC/MS) or by ElectroSpray-Ionisation tandem Mass Spectrometry (ESI-MS/MS). GAI patients with normal C5DC levels can be diagnosed by the presence of increased levels of urinary 3-OH-GA which is considered being the most confidential diagnostic marker. In some patients, the urinary excretion of GA can be completely normal and the glutaconic acid may appear during an acute attack only. Due to limited efflux across the bloodbrain barrier, the in vivo metabolic monitoring of intracerebrally accumulated neurotoxic GA and 3-OH-GA was not possible. At present, for this purpose (1)H magnetic resonance spectroscopy with the metabolite findings correlating with biochemical phenotype and duration of the disease can be applied (34). 
631-638

Also, it is necessary to consider the possibility of incorrect diagnosis that could be caused by glutaric aciduria type II (multiple acyl-CoA dehydrogenase deficiency), pseudoglutarylcarnitinaemia (caused by interfering acylcarnitines identified as hydroxyoctanoylcarnitine and hydroxydecanoylcarnitine) in patients with medium-chain acyl-CoA dehydrogenase (MCAD) deficiency as well as in newborns with renal failure, and maternal aciduria glutaric type I (17).

GAI is a treatable disorder and the guidelines for the GAI management were published by Kölker et al (16) and afterwards summarised by Heringer et al (21). Appropriate therapeutic recommendations can prevent the movement disorder and irreversible cerebral damage in GAI patients identified especially by NBS. A progressive disease with late diagnostics may lead to the development of dementia, cerebral neoplasms, peripheral neuropathy as well as chronic renal failure (19). The dietary lysine restriction leads to the reduction of accumulated GA, 3-OH-GA and glutarylCoA also in the brain. The supplementation by carnitine avert the risk of secondary carnitine depletion, increases the production of nontoxic C5DC and the adequate concentration of free carnitine in brain, liver and plasma fills up the pool of intracellular free coenzyme A. Riboflavin is administered less frequently because there is no general evidence that it improves the neurological outcome (5). Infection, fever, vomiting and/or inadequate energy intake may lead to the development of the metabolic encephalophatic crisis, which can cause an irreversible neurological damage. Thus, the emergency situations consist of the administration of highcalorie and low-protein treatment $(21,35,36)$. It is important to emphasise the fact that a neuropathic crisis in low excretors may occur independently of the patient's metabolic status, though the occurrence of these damaging episodes decreases with the age, especially after five years of life.

Biochemical phenotype in both reported patients was characterised by abnormal excretion of GA, but with different magnitude of excretion. In the first patient, monitored levels of GA were often extremely elevated even during the well compensated period and did never reach the physiological values. Thus, the disease had been manifested in a manner typical for high excretors. In the second newborn patient, the excretion of GA was only slightly increased at the time of diagnostics and after carnitine supplementation and normalisation of carnitine value in the serum, the excretion of GA was even within a normal range $(4.2 \mathrm{mmol} / \mathrm{mol}$ creatinine $)$. For these reasons, we think that the second patient could be categorised as a low excretor probably with a higher residual enzyme activity. In this case, the biochemical phenotype can result from mild pathogenic mutations found in the GCDH gene. Before carnitine supplementation, we observed low levels of free carnitine as a result of secondary depletion in both patients. In addition to an increased level of 3-OH-GA, which is most likely produced in minor pathway from glutaryl-CoA, we also observed a markedly elevated level of 2-oxoglutaric acid, aconitic acid and mildly elevated excretion of other dicarboxylic acids in both patients.

The experimental evidence exists that accumulation of GA and its metabolites may cause complex changes in metabolism due to the inhibitory effect on enzymes and transporters of dicarboxylic acids that may affect energy metabolism including the Krebs cycle (37). These effects in the central nervous system can significantly influence the constituents and mechanisms of neurotransmission. The severe form of the disease in the first patient is also supported by the finding of uncompensated metabolic acidosis, considerable hypoglycaemia and signs of hepatotoxic damage. During the follow up in the second patient, no extreme changes in the acidobasic parameters and blood glucose were observed.

In enzymopathies, the character of sequence changes may often predict a degree of enzymatic deficiency and the biochemical phenotype at least. Therefore, to predict the possible impact of sequence variants on GCDH function it is important to have knowledge about its tertiary structure. GCDH gene encodes 438 amino acids and 44 amino-terminal signal residues are cleaved off after import into the mitochondrion. The active GCDH acts as homotetramer and the monomer unit consists of three domains, two $\alpha$-helical amino-terminal domains (A - residues 45-167 and C - residues 282-438) and a $\beta$-sheet domain in the middle (B - residues 168-281). FAD is also involved in this enzymatic reaction as a coenzyme (38). Region A consists of six $\alpha$-helices and probably is not involved in the binding of substrate and except of the first five amino acid residues does not participate on oligomerisation as well. Region B is a $\beta$-sandwich, which is in the proximity with FAD coenzyme. The region C consists of four antiparallel $\alpha$-helices directly participating in the interaction with FAD by connecting loops between them. Functionally, this region represents the most important component because it creates the core of the tetramer form of the GCDH protein and the presence of pathogenic variants in the region $\mathrm{C}$ are supposed to affect the binding of FAD and contacting between monomeric units. Moreover, the amino acids sequence is evolutionary highly conserved in mouse, human and pig $\operatorname{GCDH}(38,39)$.

Besides a described mutation (c. $1262 \mathrm{C}>\mathrm{T}$ ) we also report two novel sequence variants in the GCDH gene not reported yet - a duplicating insertion c.1213dupA leading to the premature stop codon formation and a missense variant c. $1225 \mathrm{G}>\mathrm{A}$ (p.Ala409Thr) consider to be probably deleterious according to MutationTaster prediction software.

The known pathogenic variant c. $1262 \mathrm{C}>\mathrm{T}$ (p.Ala421 Val) is typical, but not restricted to the Amish community and leads to an impaired association of enzyme subunits (40). Its experimental expression in the prokaryotic system led to significant residual enzyme activity (40\%) and was termed as "leaky" pathogenic variant $(7,41)$.

In conclusion, we have identified two Slovak patients with different clinical and biochemical phenotype. Their diagnosis was confirmed at biochemical and molecular-genetic level, and two novel sequence variants in the GCDH gene had been reported. Based on the tertiary structure of the protein and the function of its particular regions, we consider these changes to be pathogenic mutations. Based on these findings, it is important to emphasise the benefit of GAI inclusion into the NBS programme arising from the possibility to express a suspicion for this neurometabolic disease in asymptomatic newborns with subsequent confirmation of diagnosis and the initiation of early therapeutic intervention 
before the symptoms or even irreversible neurological damage develop. Despite of these unequivocal positive aspects, GAI is incorporated into the NBS programmes not even in a half of E.U. member states. This decision probably arose from the fact that the disease did not fulfil all established newborn screening criteria. The incidence of GAI is extremely rare in the general population even with a relatively high proportion of asymptomatic individuals. Although the treatment can be administered in these clinically healthy patients soon after birth, its benefit is not clear. During the last twenty years only three patients were identified in Slovakia. Moreover, likewise as in our patients, the significance of novel sequence variants in the $G C D H$ gene can be ambiguous and their genotype-phenotype correlation is problematic. All these pros and cons aspects raise the question whether the inclusion of GAI into NBS is really justified in Slovakia.

\section{References}

1. Goodman SI, Markey SP, Moe PG, Miles BS, Teng CC. Glutaric aciduria; a "new" disorder of amino acid metabolism. Biochem Med 1975; 12 (1): 12-21.

2. Greenberg CR, Duncan AM, Gregory CA, Singal R, Goodman SI. Assignment of human glutaryl-CoA dehydrogenase gene (GCDH) to the short arm of chromosome 19 (19p13.2) by in situ hybridization and somatic cell hybrid analysis. Genomics 1994; 21 (1): 289-290.

3. Christensen E, Brandt NJ. Studies on glutaryl-CoA dehydrogenase in leucocytes, fibroblasts and amniotic fluid cells. The normal enzyme and the mutant form in patients with glutaric aciduria. Clin Chim Acta 1978; 88 (2): $267-276$.

4. Goodman SI, Gallegos DA, Pullin CJ et al. Antenatal diagnosis of glutaric acidemia. Am J Hum Genet 1980; 32 (5): 695-699.

5. Kölker S, Christensen E, Leonard JV et al. Diagnosis and management of glutaric aciduria type I--revised recommendations. J Inherit Metab Dis 2011; 34 (3): 677-694.

6. Kim HS, Yu HJ, Lee J et al. A Korean patient with glutaric aciduria type 1 with a novel mutation in the glutaryl CoA dehydrogenase gene. Ann Clin Lab Sci 2014; 44 (2): 213-216.

7. Hedlund GL, Longo N, Pasquali M. Glutaric acidemia type 1. Am J Med Genet C Semin Med Genet 2006; 142C (2): 86-94.

8. Gupta N, Singh PK, Kumar M et al. Glutaric Acidemia Type 1-ClinicoMolecular Profile and Novel Mutations in GCDH Gene in Indian Patients. JIMD Rep 2015; 21: 45-55.

9. Baric I, Wagner L, Feyh P, Liesert M, Buckel W, Hoffmann GF. Sensitivity and specificity of free and total glutaric acid and 3-hydroxyglutaric acid measurements by stable-isotope dilution assays for the diagnosis of glutaric aciduria type I. J Inherit Metab Dis 1999; 22 (8): 867-881.

10. Wang Q, Yang YL. Complex heterogeneity phenotypes and genotypes of glutaric aciduria type 1. Zhongguo Dang Dai Er Ke Za Zhi 2016; 18 (5): 460-465.

11. Afroze B, Yunus ZM. Glutaric aciduria type 1 - importance of early diagnosis and treatment. J Pak Med Assoc 2014; 64 (5): 593-595.

12. Boy N, Heringer J, Haege G et al. A cross-sectional controlled developmental study of neuropsychological functions in patients with glutaric aciduria type I. Orphanet J Rare Dis 2015; 10: 163.
13. Kyllerman M., Skjeldal OH, Lundberg M et al. Dystonia and dyskinesia in glutaric aciduria type I: clinical heterogeneity and therapeutic considerations. Mov Disord 1994; 9 (1): 22-30.

14. Mohamed S, Hamad MH, Hassan HH, Salih MA. Glutaric aciduria type 1 as a cause of dystonic cerebral palsy. Saudi Med J 2015; 36 (11): 1354-1357.

15. Vester ME, Visser G, Wijburg FA, van Spronsen FJ, Williams M, van Rijn RR. Occurrence of subdural hematomas in Dutch glutaric aciduria type 1 patients. Eur J Pediatr 2016; 175 (7): 1001-1006.

16. Kölker S, Christensen E, Leonard JV et al. Guideline for the diagnosis and management of glutaryl-CoA dehydrogenase deficiency (glutaric aciduria type I). J Inherit Metab Dis 2007; 30 (1): 5-22.

17. Heringer J, Boy N, Burgard P, Okun JG, Kölker S. Newborn Screening for Glutaric Aciduria Type I: Benefits and limitations. Int J Neonat Screen 2015; 1 (2): 57-68.

18. Radha Rama Devi A, Ramesh VA, Nagarajaram HA, Satish SP, Jayanthi U, Lingappa L. Spectrum of mutations in Glutaryl-CoA dehydrogenase gene in glutaric aciduria type I-Study from South India. Brain Dev 2016; 38 (1): 54-60.

19. Heringer $\mathbf{J}$, Valayannopoulos $\mathbf{V}$, Lund $\mathbf{A M}$ et al. Impact of age at onset and newborn screening on outcome in organic acidurias. J Inherit Metab Dis 2016; 39 (3): 341-353.

20. Rinaldo P. Organic acids. 137-171. In: Blau N, Duran M, Gibson KM (Eds). Laboratory Guide to the Methods in Biochemical Genetics. Verlag Berlin Heidelberg: Springer, 2008.

21. Heringer J, Boy SP, Ensenauer R et al. Use of guidelines improves the neurological outcome in glutaric aciduria type I. Ann Neurol 2010; 68 (5): 743-752.

22. Lindner M, Kölker S, Schulze A, Christensen E, Greenberg CR, Hoffmann GF. Neonatal screening for glutaryl-CoA dehydrogenase deficiency. J Inherit Metab Dis 2004; 27 (6): 851-859.

23. Pfeil J, Listl S, Hoffmann GF, Kölker S, Lindner M, Burgard P. Newborn screening by tandem mass spectrometry for glutaric aciduria type 1: a cost-effectiveness analysis. Orphanet J Rare Dis 2013; 8: 167.

24. Bähr O, Mader I, Zschocke J, Dichgans J, Schulz JB. Adult onset glutaric aciduria type I presenting with a leukoencephalopathy. Neurology 2002; 59 (11): 1802-1804.

25. Külkens S, Harting I, Sauer $\mathbf{S}$ et al. Late-onset neurologic disease in glutaryl-CoA dehydrogenase deficiency. Neurology 2005; 64 (12): 2142-2144.

26. Herskovitz M, Goldsher D, Sela BA, Mandel H. Subependymal mass lesions and peripheral polyneuropathy in adult-onset glutaric aciduria type I. Neurology 2013; 81 (9): 849-850.

27. Pierson TM, Nezhad M, Tremblay MA et al. Adult-onset glutaric aciduria type I presenting with white matter abnormalities and subependymal nodules. Neurogenetics 2015; 16 (4): 325-328.

28. Badve MS, Bhuta S, Mcgill J. Rare presentation of a treatable disorder: glutaric aciduria type 1. N Z Med J 2015; 128 (1409): 61-64.

29. Korman SH, Jakobs C, Darmin PS et al. Glutaric aciduria type 1: clinical, biochemical and molecular findings in patients from Israel. Eur J Paediatr Neurol 2007; 11 (2): 81-89.

30. Zhang Y, Li H, Ma $\mathbf{R}$ et al. Clinical and molecular investigation in Chinese patients with glutaric aciduria type I. Clin Chim Acta 2016; 453: 75-79. 


\section{1-638}

31. Bessey A, Chilcott J, Pandor A, Paisley S. The Cost-Effectiveness of Expanding the Nhs Newborn Bloodspot Screening Programme To Include Homocystinuria (Hcu), Maple Syrup Urine Disease (Msud), Glutaric Aciduria Type 1 (Ga1), Isovaleric Acidaemia (Iva), and Long-Chain Hydroxyacyl-Coa Dehydrogenase Deficiency (Lchadd). Value Health 2014; 17 (7): A531.

32. Babu RP, Bishnupriya G, Thushara PK et al. Detection of glutaric acidemia type 1 in infants through tandem mass spectrometry. Mol Genet Metab Rep 2015; 3: 75-79.

33. El-Mesellamy H, Gouda AS, Fateen E, Zaki SS. Disorders associated with abnormal acylcarnitine profile among high risk Egyptian children. Bratisl Lek Listy 2014; 115 (5): 300-306.

34. Harting I, Boy N, Heringer $\mathbf{J}$ et al. (1)H-MRS in glutaric aciduria type 1: impact of biochemical phenotype and age on the cerebral accumulation of neurotoxic metabolites. J Inherit Metab Dis 2015; 38 (5): $829-838$

35. Zinnanti WJ, Lazovic J, Housman C et al. Mechanism of age-dependent susceptibility and novel treatment strategy in glutaric acidemia type I. J Clin Invest 2007; 117 (11): 3258-3270.
36. Sauer SW, Opp S, Hoffmann GF, Koeller DM, Okun JG, Kölker S. Therapeutic modulation of cerebral L-lysine metabolism in a mouse model for glutaric aciduria type I. Brain 2011; 134 (Pt 1): 157-170.

37. Lamp J, Keyser B, Koeller DM, Ullrich K, Braulke T, Mühlhausen C. Glutaric aciduria type 1 metabolites impair the succinate transport from astrocytic to neuronal cells. J Biol Chem 2011; 286 (20): 17777-17784.

38. Busquets $\mathbf{C}$, Merinero B, Christensen $\mathbf{E}$ et al. Glutaryl-CoA dehydrogenase deficiency in Spain: evidence of two groups of patients, genetically, and biochemically distinct. Pediatr Res 2000; 48 (3): 315-322.

39. Koeller DM, DiGiulio KA, Angeloni SV et al. Cloning, structure, and chromosome localization of the mouse glutaryl-CoA dehydrogenase gene. Genomics 1995; 28 (3): 508-512.

40. Biery BJ, Stein DE, Morton DH, Goodman SI. Gene structure and mutations of glutaryl-coenzyme A dehydrogenase: impaired association of enzyme subunits that is due to an A421V substitution causes glutaric acidemia type I in the Amish. Am J Hum Genet 1996; 59 (5): 1006-1011.

41. Fraidakis MJ, Liadinioti C, Stefanis L et al. Rare Late-Onset Presentation of Glutaric Aciduria Type I in a 16-Year-Old Woman with a Novel GCDH Mutation. JIMD Rep 2015; 18: 85-92.

Received May 18, 2016. Accepted August 8, 2016. 